\title{
Neutrophil Activation and Resistance to Recombinant Human Erythropoietin Therapy in Hemodialysis Patients
}

\author{
Elísio Costa ${ }^{a-c}$ Susana Rocha ${ }^{a, b}$ Petronila Rocha-Pereira ${ }^{b, d}$ \\ Henrique Nascimento ${ }^{a, b}$ Elisabeth Castro ${ }^{a, b}$ Vasco Miranda $^{e}$ \\ Maria do Sameiro Faria ${ }^{e}$ Alfredo Loureirof Alexandre Quintanilha ${ }^{b, g}$ \\ Luis Belo ${ }^{a, b}$ Alice Santos-Silva ${ }^{a, b}$ \\ a Faculdade Farmácia, Serviço de Bioquimica, Universidade do Porto, Porto; b' Instituto Biologia Molecular e \\ Celular, Universidade do Porto, Porto; 'Instituto de Ciências da Saúde, Centro Regional do Porto da Universidade \\ Católica Portuguesa, Porto; ' ${ }^{\mathrm{C}}$ Centro de Investigação em Ciências da Saúde, Universidade Beira Interior, Covilhā; \\ 'EMC, Dinefro - Diálises e Nefrologia, SA, Maia; f Uninefro - Sociedade Prestadora de Cuidados Médicos e de Diálise, \\ SA, Santo Tirso; 9Instituto Ciências Biomédicas Abel Salazar, Universidade do Porto, Porto, Portugal
}

\section{Key Words}

rhEPO therapy, resistance $\cdot$ Neutrophil activation $\cdot$ Elastase $\cdot$ Lactoferrin

\footnotetext{
Abstract

Aim: The aim of this work was to evaluate the neutrophil activation state in chronic kidney disease (CKD) patients under hemodialysis, and its linkage with resistance to recombinant human erythropoietin (rhEPO) therapy. Methods: We studied 63 CKD patients under hemodialysis and rhEPO treatment $(32$ responders and 31 non-responders to rhEPO therapy). In 20 of the CKD patients (10 responders and 10 non-responders to rhEPO therapy), blood samples were also collected immediately after dialysis. Twenty-six healthy volunteers were included in a control group. Hemoglobin levels, total and differential leukocyte counts, and circulating levels of C-reactive protein (CRP), elastase and lactoferrin were measured in all patients and controls. Results: Com-
}

pared with controls, CKD patients presented with significantly higher CRP, neutrophil and elastase levels. When we compared the 2 groups of patients, we found that nonresponders presented statistically significantly higher elastase plasma levels. A positive significant correlation was found between elastase levels and weekly rhEPO dose and CRP serum levels. After the hemodialysis procedure, a statistically significant rise in elastase, lactoferrin and, elastase/ neutrophil and lactoferrin/neutrophil ratios were found. Conclusions: Our data show that CKD patients under hemodialysis present higher elastase levels (particularly in nonresponding patients), which could be related to the rise in neutrophils, and to be part of the enhanced inflammatory process found in these patients. 


\section{Introduction}

Despite the technologic developments in hemodialysis procedures and medical support over the last years, the mortality and morbidity of chronic kidney disease (CKD) patients under hemodialysis remains high, about 10-20 times higher than that found in the general population. Anemia is the most frequent complication associated with hemodialysis [1-3].

The management of anemia was improved by the introduction of recombinant human erythropoietin (rhEPO) therapy, allowing a significant correction of anemia. However, there is marked variability in the sensitivity to rhEPO, with an up to 10 -fold variability in dose requirements to achieve correction of anemia, and around 5$10 \%$ of the patients showing a marked resistance to rhEPO therapy [4-6].

The interaction of blood with non-biological materials of the extracorporeal circuit during hemodialysis leads to the activation of several non-cellular. and cellular systems, including polymorphonuclear leukocytes (PMN) $[7,8]$. The activation of PMN may lead to degranulation, with release of several components, namely, cationic proteins and proteases and the production of oxygen metabolites. Reports on this subject $[7,9,10]$, though in a limited number, have shown that neutrophils are activated during the hemodialysis procedure, but none of those reports studied the relationship between resistance to rhEPO therapy and neutrophil activation markers. Actually, it has been reported that rhEPO resistance seems to be an associated inflammatory feature. Moreover, none of those works calculated the ratios of neutrophil activation products per number of neutrophils, a more accurate measure of leukocyte activation.

The aim of this work was to evaluate the neutrophil activation state in CKD patients under hemodialysis, and its linkage with resistance to rhEPO therapy by measuring circulating levels of elastase and lactoferrin. These two substances are contained within primary and secondary neutrophil granules, respectively, and are frequently used as indirect markers of neutrophil activation in vivo.

\section{Subjects and Methods}

We performed a cross-sectional study by evaluating 63 CKD patients under hemodialysis ( 36 males, 27 females; mean age \pm SD $62.1 \pm 15.7$ years) and rhEPO treatment. The CKD patients included 32 responders and 31 non-responders to rhEPO therapy. Classification of the patients as responders or non-responders was performed in accordance with the European Best Practice Guidelines [11] which defines resistance to rhEPO as failure to achieve target hemoglobin levels (between 11 and $12 \mathrm{~g} / \mathrm{dl}$ ) with maintenance doses of $\mathrm{rhEPO}$ of $>300 \mathrm{JU} / \mathrm{kg} /$ week of epoetin or $1.5 \mu \mathrm{g} /$ $\mathrm{kg} /$ week of darbepoietin- $\alpha$. The rhEPO maintenance dose for responding patients was $89.65 \pm 57.62 \mathrm{U} / \mathrm{kg} /$ week and for non-responders $572.99 \pm 193.84 \mathrm{U} / \mathrm{kg} /$ week. The 2 groups of patients were matched for age (responders $62.3 \pm 16.8$ years vs. non-responders $62.9 \pm 15.1$ years, $p>0.05$ ), gender (responders $56.25 \%$ males vs. non-responders $58.06 \%$ males, $p>0.05$ ), weight (responders $63.9 \pm 11.7 \mathrm{~kg}$ vs. non-responders $58.6 \pm 13.1 \mathrm{~kg} ; \mathrm{p}>$ 0.05 ), body mass index (responders $23.7 \pm 3.4 \mathrm{vs}$. non-responders $21.8 \pm 4.7, p>0.05$ ), mean time on hemodialysis (responders 68.0 \pm 65.7 months vs. non-responders $62.88 \pm 51.2$ months, $p>$ 0.05 ), urea reduction ratio (responders $25.58 \pm 7.71 \%$ vs. non-responders $27.8 \pm 8.6 \%, p>0.05$ ), urea Ktv (responders $1.5 \pm 0.3$ vs. non-responders $1.63 \pm 0.3, p>0.05$ ) and parathyroid hormone serum levels (responders $294.5 \pm 282.9 \mathrm{pg} / \mathrm{ml}$ vs. non-responders $282.2 \pm 244.7 \mathrm{pg} / \mathrm{ml}, \mathrm{p}>0.05$ ).

Twenty of the CKD patients (10 responders and 10 non-responders to rhEPO therapy) were also evaluated before and immediately after hemodialysis.

CKD patients were under therapeutic hemodialysis three times per week, for 3-5 h, for a median of 36 months. All patients used the high-flux polysulfone FX-class dialyzers of Fresenius: 34 with FX60;27 with FX80, and 2 with FX100 dialyzer type. The causes of renal failure in the patient population were as follows: diabetic nephropathy $(n=19)$; chronic glomerulonephritis $(n=$ 11); polycystic kidney disease $(n=3)$; hypertensive nephrosclerosis $(n=3)$; obstructive nephropathy $(n=3)$; pyelonephritis associated with neurogenic bladder $(n=1)$; nephrolithiasis $(n=1)$; chronic interstitial nephritis $(n=1)$; Alport syndrome $(n=1)$; renal vascular disease due to polyarteritis $(n=1)$, and chronic renal failure of uncertain etiology $(n=19)$. Patients with autoimmune diseases, malignancy, hematological disorders, and acute or chronic infection were excluded. Intravenous iron supplementation was based on the European Best Practice Guidelines for the management of anemia in patients with hemodialysis [11]. All patients gave their informed consent to participate in this study.

Healthy volunteer were selected as controls based on normal hematological and biochemical values, and no history of kidney or inflammatory disease. They were also matched as far as possible for age and gender with the CKD patients (8 males, $17 \mathrm{fe}$ males; mean $\pm S D$ age $47.81 \pm 14.69$ years).

\section{Blood Sample Assays}

Blood samples were collected with and without EDTA as anticoagulant in order to obtain whole blood, serum and plasma. In the cross-sectional study, blood samples were collected from CKD patients before starting hemodialysis. To evaluate the effect of hemodialysis in the parameters studied, blood samples were also collected immediately after hemodialysis.

The hemoglobin concentration and white blood cell count were measured using an automatic counter (Sysmex K1000, Hamburg, Germany) and leukocyte differential counts were evaluated in Wright-stained blood films. Plasma levels of elastase and lactoferrin were evaluated by enzyme immunoassays (human PMN Elastase ELISA, Bender MedSystems; Lactoferrin ELISA Kit, Calbiochem, respectively). Serum C-reactive protein (CRP) was determined by immunoturbidimetry (CRP latex HS Roche kit, 
Table 1. Hematological data, iron status and neutrophil activation markers for controls and CKD patients

\begin{tabular}{|c|c|c|c|c|}
\hline & Controls $(n=26)$ & All patients ( $n=63$ ) & Responders $(n=32)$ & Non-responders $(n=31)$ \\
\hline $\mathrm{Hb}, \mathrm{g} / \mathrm{dl}$ & $13.90(13.2-15.00)$ & $10.90(10.30-12.30)^{a}$ & $11.70(10.83-12.68)^{2}$ & $10.4(9.00-11.30)^{a . b}$ \\
\hline White cell counts, $\times 10^{4} / 1$ & $5.78 \pm 1.59$ & $6.23 \pm 2.10$ & $6.42 \pm 1.96$ & $6.04 \pm 2.26$ \\
\hline Lymphocyt'es, $\times 10^{9} / 1$ & $2.35 \pm 0.75$ & $1.47 \pm 0.60^{\mathrm{a}}$ & $1.58 \pm 0.49^{a}$ & $1.36 \pm 0.69^{\mathrm{a}, \mathrm{b}}$ \\
\hline Monocytes, $\times 10^{9} / 1$ & $0.25 \pm 0.08$ & $0.38 \pm 0.16^{\mathrm{a}}$ & $0.40 \pm 0.13^{a}$ & $0.35 \pm 0.17^{a}$ \\
\hline Neutrophils, $\times 10^{9} / 1$ & $3.03 \pm 1.02$ & $4.14 \pm 1.79^{2}$ & $4.17 \pm 1.87^{\mathrm{a}}$ & $4.11 \pm 1.73^{\mathrm{a}}$ \\
\hline Ferritin, $\mathrm{ng} / \mathrm{ml}$ & $85.10(40.90-123.00)$ & $383.80(188.20-546.25)^{b}$ & $399.00(294.68-545.25)^{b}$ & $342.00(163.00-580.05)^{b}$ \\
\hline Tranferrin saturation, $\%$ & $19.44(17.28-27.94)$ & $21.80(17.70-30.91)$ & $24.20(19.96-33.29)$ & $22.10(17.79-29.44)$ \\
\hline Albumin, g/dl & NM & $3.8 \pm 0.4$ & $4.0 \pm 0.4$ & $3.7 \pm 0.4^{b}$ \\
\hline $\mathrm{CRP}, \mathrm{mg} / \mathrm{dl}$ & $1.75(0.76-4.70)$ & $5.75(1.90-14.01)^{\mathrm{a}}$ & $3.20(1.73-7.23)^{\mathrm{a}}$ & $10.14(3.82-38.99)^{a . b}$ \\
\hline Elastase, $\mu \mathrm{g} / \mathrm{l}$ & $28.29(26.03-34.74)$ & $36.11(29.69-50.65)^{4}$ & $34.13(28.76-39.16)^{a}$ & $39.75(31.15-64.84)^{a, b}$ \\
\hline Elastase/neutrophil ratio & $10.86(7.44-12.12)$ & $8.91(7.43-13.78)$ & $8.70(7.32-11.42)$ & $10.25(7.56-17.41)$ \\
\hline Lactoferrin, $\mu \mathrm{g} / 1$ & $236.56(193.56-295.03)$ & $239.35(165.64-332.60)$ & $239.60(170.90-332.70)$ & $228.90(160.33-341.50)$ \\
\hline Lactoferrin/neutrophil ratio & $72.11(55.52-111.83)$ & $60.32(42.82-99.45)$ & $64.55(44.31-101.49)$ & $58.78(41.50-102.99)$ \\
\hline
\end{tabular}

$\mathrm{NM}=$ Not made. Results are presented as mean \pm standard deviation and as median (interquartile ranges).

${ }^{\mathrm{a}} \mathrm{p}<0.05$ vs. controls; ${ }^{\mathrm{b}} \mathrm{p}<0.05 \mathrm{vs}$. responders.

Roche Diagnostics). Serum albumin levels were measured using a colorimetric assay end-point method (Albumin Plus; Roche $\mathrm{GmbH}$, Mannheim, Germany). The serum iron concentration was determined using a colorimetric method (Randox Laboratories Ltd., Northern Ireland, UK), whereas serum ferritin (Randox Laboratories Ltd.) and serum transferrin (Randox Laboratories Ltd.) were measured by immunoturbidimetry. Transferrin saturation (TS) was calculated using the formula: TS $(\%)=70.9 \times$ serum iron concentration $(\mu \mathrm{g} / \mathrm{dl}) /$ serum transferrin concentration (mg/dl).

\section{Data Analysis}

For statistical analysis, we used the Statistical Package for Social Sciences (SPSS version 15.0 for Windows, SPSS Inc., Chicago, Ill., USA). Kolmogorov Smirnov statistics were used to evaluate sample normality distribution. Multiple comparisons between groups were performed by one-way ANOVA supplemented with Turkey's HSD post hoc test. For single comparisons, we used the Student's t test whenever the parameters presented a Gaussian distribution and the Mann-Whitney $U$ test in the case of a nonGaussian distribution. To compare data before and after hemodialysis, we used paired-samples t test or Wilcoxon test. Spearman's rank correlation coefficient was used to evaluate relationships between sets of data. Significance was accepted at $p<0.05$.

\section{Results}

The hematological characteristics, iron status and the inflammatory markers of the 3 groups studied (crosssectional study) are summarized in table 1 . Hemodialysis $\mathrm{CKD}$ patients were anemic, with non-responding patients being more anemic than responders. No difference was found between controls and patients concerning the white blood cell count. However, CKD patients showed lymphopenia, as well as higher monocyte and neutrophil counts as compared to controls. When comparing the 2 groups of patients, non-responders presented a significantly lower lymphocyte count than responders.

Compared with controls, CKD patients presented significantly higher ferritin, CRP and elastase plasma levels (table 2). However, no statistically significant differences were found for the elastase/neutrophil ratio. No statistically significant differences were also found between CKD patients and controls for plasma levels of lactoferrin and lactoferrin/neutrophil ratio. When we compared the 2 groups of patients, we found that non-responders showed decreased serum levels of albumin, and higher CRP and elastase plasma levels. Statistically significantly positive correlations were found between elastase plasma levels and weekly rhEPO dose and CRP (fig. 1).

Analyzing the results for hematological data, and for neutrophil activation markers, before and immediately after the hemodialysis procedure (table 2), we found a statistically significant rise in hemoglobin, elastase, lactoferrin and, elastase/neutrophil and lactoferrin/neutrophil ratios. No statistically significant changes were found between responders and non-responders for any of the parameters studied.

\section{Discussion}

As far as we know, we are the first group to assess the association between neutrophil activation and resistance to rhEPO therapy in CKD patients under hemodialysis. 

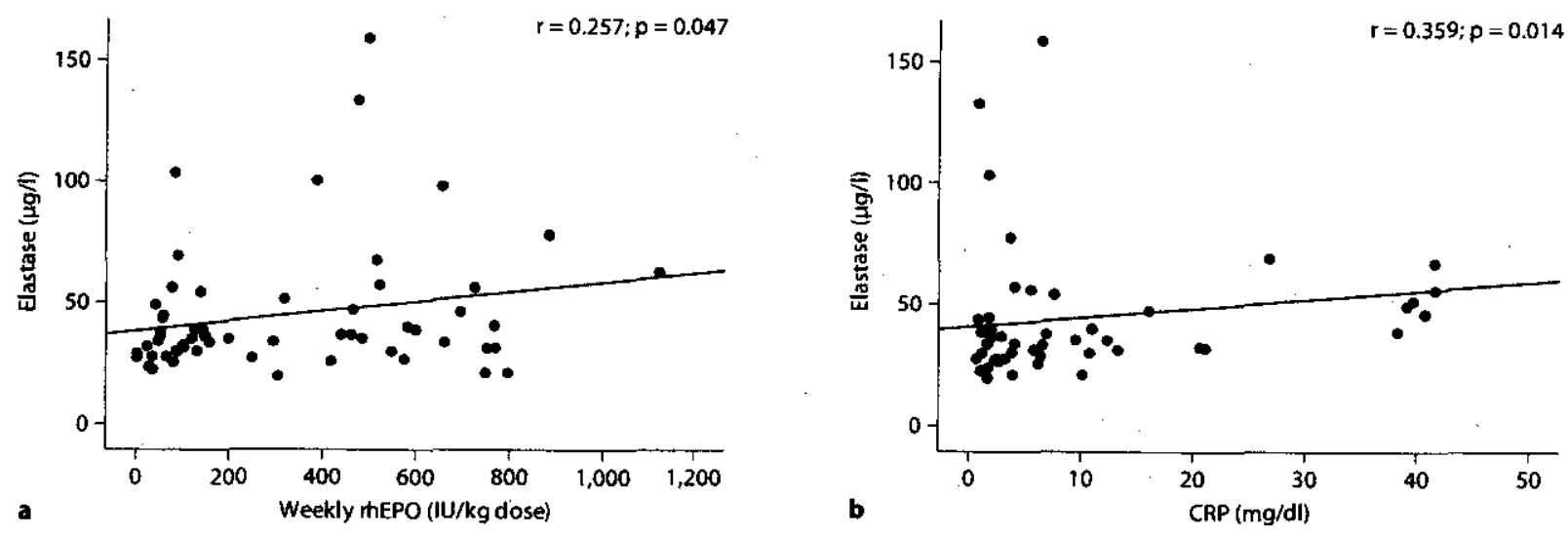

Fig. 1. Correlation between plasma elastase levels and weekly rhEPO dose (a) and CRP (b) in hemodialysis patients.

Table 2. Hematological data and neutrophil activation markers for CKD patients, before and after the hemodialysis procedure

\begin{tabular}{lcc}
\hline & \multicolumn{2}{l}{ Hemodialysis patients $(\mathbf{n}=20)$} \\
\cline { 2 - 3 } & \multicolumn{1}{l}{ before } & after \\
\hline $\mathrm{Hb}, \mathrm{g} / \mathrm{dl}$ & $12.10(10.95-12.80)$ & $13.20(11.15-14.60)^{\mathrm{a}}$ \\
White cell counts, $\times 10^{9} / 1$ & $5.86 \pm 1.51$ & $5.93 \pm 2.19$ \\
Neutrophils, $\times 10^{9} / 1$ & $3.82 \pm 1.24$ & $3.97 \pm 1.77$ \\
Monocytes, $\times 10^{9} / \mathrm{l}$ & $0.24 \pm 0.38$ & $0.17 \pm 0.12$ \\
Lymphocytes, $\times 10^{9} / \mathrm{l}$ & $1.64 \pm 0.69$ & $1.66 \pm 0.64$ \\
Elastase, $\mu \mathrm{g} / \mathrm{l}$ & $36.16(29.71-47.13)$ & $51.69(40.08-71.68)^{\mathrm{a}}$ \\
Elastase/neutrophil ratio & $10.66(7.32-13.54)$ & $14.66(13.34-18.95)^{\mathrm{a}}$ \\
Lactoferrin, $\mu \mathrm{g} / \mathrm{l}$ & $198.61(137.81-216.97)$ & $236.56(171.28-363.63)^{\mathrm{a}}$ \\
Lactoferrin/neutrophil ratio & $48.33(33.88-64.31)$ & $60.72(51.81-94.81)^{\mathrm{a}}$ \\
$\mathrm{CRP}, \mathrm{mg} / \mathrm{dl}$ & $3.06(1.39-5.22)$ & $3.53(1.54-5.56)$ \\
\hline
\end{tabular}

Results are presented as mean \pm standard deviation and as median (interquartile ranges). ${ }^{a} \mathrm{p}<0.05 \mathrm{vs}$. before hemodialysis.
We were able to demonstrate that elastase, a neutrophil protease released by degranulation, is significantly higher in the plasma of CKD patients under hemodialysis, being particularly high in non-responding CKD patients. This is probably part of the enhanced inflammatory status reported for these CKD patients.

In the cross-sectional study, performed before hemodialysis, we assessed chronic inflammation associated with CKD and with resistance to rhEPO therapy. We found that CKD patients under hemodialysis treatment show a significant rise in neutrophils and increased CRP levels in serum, confirming the presence of an inflamma- tory process in these patients. The fact that non-responders had higher CRP levels suggests that inflammation is related to resistance to rhEPO therapy, in agreement with previous studies $[12,13]$. The lymphopenia found in hemodialysis patients is particularly enhanced in non-responders. This feature has already been described in the literature [13] and results, at least in part, from a decrease in total circulating CD3 $+\mathrm{T}$ lymphocytes and affects both the CD4+ and the CD8+ T-cell subsets.

We used lactoferrin and elastase plasma levels as neutrophil activation markers. Elastase levels were significantly higher in hemodialysis patients, particularly in 
non-responding patients. However, no statistical differences were found for the elastase/neutrophil ratio between controls and patient groups, suggesting that the higher elastase levels are mainly associated with the increased neutrophil count found in these patients. Even so, it is important to highlight that the elastase/neutrophil ratio presented a trend to higher values in non-responders. Moreover, we found statistically significant positive correlations between elastase levels and CRP, suggesting that the rise in elastase levels is part of the inflammatory process found in hemodialysis patients and particularly enhanced in non-responders. The statistically significant correlation between elastase levels and weekly rhEPO doses also corroborate this hypothesis; in fact, non-responders to rhEPO therapy who require higher weekly rhEPO doses to achieve target hemoglobin levels are also associated with an increased inflammatory process.

On the other hand, plasma levels of lactoferrin and the lactoferrin/neutrophil ratio did not differ between groups. A plausible justification for this result is that, although the major circulating lactoferrin is neutrophil-derived, it may also be produced by other cells [15]. Contrary to the evaluation of elastase, the ELISA kit used is not specific for the evaluation of PMN leukocyte lactoferrin levels. Thus, in our study, elastase is a more specific marker of neutrophil activation. Moreover, we can also consider the hypothesis that lactoferrin is being used or cleared from the circulation in a pronounced way. Indeed, lactoferrin is involved in iron metabolism, which is known to be altered in these patients [16].

By evaluating CKD patients before and after hemodialysis, we assessed whether our patients respond in same manner to the hemodialysis process. After hemodialysis, a higher hemoglobin level was found, and has been at- tributed to a translocation of erythrocytes from the splanchnic circulation (and possibly from the spleen) in order to compensate for the hypovolemic stress during dialysis ultrafiltration $[17,18]$. After hemodialysis we also found a statistically significant increase in both elastase and lactoferrin concentrations (and in their ratios per neutrophil), suggesting a neutrophilic activation process with degranulation during the hemodialysis procedure. We also found that neutrophils from non-responding $\mathrm{pa}$ tients to rhEPO therapy do not respond any differently than those found in responding patients. It is likely that the higher elastase levels found in non-responders (crosssectional study) is likely to result from a chronic but not acute stimulus of the hemodialysis process.

In conclusion, our data show that CKD patients under hemodialysis present higher elastase levels (particularly in non-responding patients), which could be related to the rise in neutrophils and could be part of the enhanced inflammatory process found in these patients. Moreover, neutrophil activation is triggered by the hemodialysis procedure, but does not explain the higher elastase levels found in non-responding patients. Elastase, but not lactoferrin, may prove to be a good marker of resistance to rhEPO therapy in CKD patients under hemodialysis.

\section{Acknowledgements}

We are very grateful to the nurses of FMC, Dinefro - Diálises e Nefrologia, SA, and Uninefro - Sociedade Prestadora de Cuidados Médicos e de Diálise, SA, for technical support. We are also very grateful to Amgen and to Roche Farmacêutica Quimica, Lda for financial support. This study was supported by a $\mathrm{PhD}$ grant (SFRH/BD/27688/2006) to E. Costa from FCT and FSE.

\section{References}

1 Foley RN, Parfrey PS, Sarnak MJ: Clinical epidemiology of cardiovascular disease in chronic renal failure. Am J Kidney Dis 1998; 32(suppl 3):S112-S119.

2 Foley RN, Parfrey PS, Harnett JD, Kent GM, Murray DC, Barre PE: The impact of anemia on cardiomyopathy morbidity and mortality in end-stage renal disease. Am J Kidney Dis 1996;28:53-61.

3 Locatelli F, Conte F, Marcelli.D: The impact of hematocrit levels and erythropoietin treatment on overall and cardiovascular mortality and morbidity - the experience of the Lombardy dialysis registry. Nephrol Dial Transplant 1998;13:1642-1644.
4 Spittle MA, Hoenich NA, Handelman GJ, Adhikarla R, Homel P, Levin NW: Oxidative stress and inflammation in hemodialysis patients. Am J Kidney Dis 2001;38:1408-14I3.

5 Pupim LB, Himmelfarb J, McMonagle E, Shyr Y, Ikizler TA: Influence of initiation of maintenance hemodialysis on biomarkers of inflammation and oxidative stress. Kidney Int 2004;65:2371-2379.

6 Sommerburg O, Grune T, Hampl H, Riedel E, van Kujjk FJ, Ehrich JHH, Siems WG: Does long-term treatment of renal anemia with recombinant erythropoietin influence oxidative stress in haemodialysed patients? Nephrol Dial Transplant 1998;13:25832587.
7 Lucchi L, Bergamini S, Botti B, Rapanà R, Ciuffreda A, Ruggiero P, Ballestri M, Tomasi A, Albertazzi A: Influence of different hemodialysis membranes on red blood cell susceptibility to oxidative stress. Artif Organs 2000;24:1-6.

8 Valentini J, Schmitt GC, Grotto D, Santa Ma ria LD, Boeira SP, Piva SJ, Brucker N, Bohrer D, Pomblum VI, Emanuelli T, Garcia SC: Human erythrocyte delta-aminolevulinate dehydratase activity and oxidative stress in hemodialysis patients. Clin Biochem 2007; 40:591-594. 
9 Skoutelis AT, Kaleridis VE, Goumenos DS Athanassiou GM, Missirlis YF, Vlachojannis JG, Bassaris HP: Polymorphonuclear leukocyte rigidity is defective in patients with chronic renal failure. Nephrol Dial Transpiant 2000;15:1788-1793.

10 Leitienne $P$, Fouque D, Rigal D, Adeleine $P$ Trzeciak MC, Laville M: Heparins and blood polymorphonuclear stimulation in haemodialysis: an expansion of the biocompatibility concept. Nephrol Dial Transplant 2000; 15:1631-1637.

11 Locatelli F, Aljama P, Barany P, Canaud B, Carrera F, Eckardt KU, Horl WH, Macdougal IC, Macleod A, Wiecek A, Cameron S European Best Practice Guidelines Working Group: Revised European best practice guidelines for the management of anemia in patients with chronic renal failure. Nephrol Dial Transplant 2004;19(suppl 2):ii1-ii47.
12 Bárány P: Inflammation, serum C-reactive protein, and erythropoietin resistance. Nephrol Dial Transplant 2001;16:224-227.

13 Costa E, Lima M, Alves IM, Rocha S, Rocha Pereira P, Castro E, Miranda V, SameiroFaria M, Loureiro A, Quintanilha A, Belo L, Santos-Silva A: Inflammation, T-cell phenotype and inflammatory cytokines in chronic kidney disease patients under haemodialysis and its relationship to resistance to recombinant human erythropoietin therapy. / Clin Immunol 2008;28:268-275.

14 Belo L, Santos-Silva A, Caslake M, Cooney J, Pereira-Leite L, Quintanilha A, Rebelo I: Neutrophil activation and $\mathrm{C}$-reactive protein concentration in preeclampsia. Hypertens Pregnancy 2003;22:129-141.
15 Levay PF, Viljoen M: Lactoferrin: a general review. Haematologica 1995;80:252-267.

16 Costa E, Pereira BJG, Rocha-Pereira P, Rocha $S$, Reis F, Castro E, Teixeira F, Miranda V, Sameiro Faria M, Loureiro A, Quintanilha A, Belo L, Santos-Silva A: Role of prohepcidin, inflammatory markers and iron status in resistance to rhEPO therapy in hemodialysis patients. Am J Nephrol 2008;28:677683.

17 Dasselaar JI, Hooge MNL, Pruim J, Nijnuis $H$, Wiersum A, Jong PE, Huisman RM Franssen CFM: Relative blood volume changes underestimated total blood volume changes during hemodialysis. Clin J Am Soc Nephrol 2007;2:669-674.

18 Yu AW, Nawab ZM, Barnes WE, Lai KN, Ing TS, Daugirdas IT: Splanchnic erythrocyte content decreases during hemodialysis: a new compensatory mechanism for hypovolemia. Kidney Int 1997;51:1986-1990. 